\title{
Mucinous Adenocarcinoma Arising From Mature Cystic Teratoma with Nodal Metastasis
}

\author{
Dr.S.Kavitha ${ }^{1}$, Dr.M.P.Kanchana ${ }^{2}$ M.D., (Pathology), \\ Dr.Babyvasumathi ${ }^{3}$ MD DGO \\ ${ }^{I}$ II Year Post Graduate, Department Of Pathology, Madras Medical College, Chennai \\ ${ }^{2}$ Professor, Department Of Pathology, Institute Of Obstetrics And Gynaecology, Chennai \\ ${ }^{3}$ The Director, Institute Of Obstetrics And Gynaecology, Chennai
}

\begin{abstract}
The most common germ cell tumor of ovary is mature cystic teratoma (MCT). Malignant transformation occurs in 1-2\% of the cases, and most of the malignancy from the MCT are squamous cell carcinoma, representing $75 \%$ of malignant transformation. The incidence of malignant transformation of adenocarcinoma is rare about $6.8 \%$. Here we present a rare case coexistence of mucinous adenocarcinoma with benign cystic teratoma in the same ovary with omental, pelvic and para aortic node metastasis.
\end{abstract}

Keywords: Mature cystic teratoma, Mucinous adenocarcinoma, Nodal metastasis.

\section{Introduction}

The most common germ cell tumor of ovary is mature cystic teratoma composing $20 \%$ of ovarian neoplasm with peak incidence in the first two decades of life. Malignant transformation occurs in 1-2\% of all mature cystic teratoma. Squamous cell carcinoma is the most common malignant transformation. Other tumours arising from MCT are adenocarcinoma, basal cell carcinoma, adeno squamous carcinoma, thyroid carcinoma, sebaceous carcinoma, malignant melanoma, sarcoma, carcinoid tumour and neuro ectodermal tumour. We present a case of mucinous cyst adenocarcinoma arising from MCT with omental, pelvic, and para aortic metastasis

\section{Case Report}

A 44 year old female patient $\left(\mathrm{P}_{1} \mathrm{~L}_{1} \mathrm{~A}_{1}\right)$ presented to the emergency unit with history of abdominal distension for 2 months associated with intermittent abdominal pain. There was no history of dysmennorhoea, menorrhagia, bowel and bladder disturbances. Per abdomen revealed solid hard mass measuring 10x8 cm occupying all quadrants below umbilicus with restricted mobility and lower border not made out. Ultrasonogram abdomen shows a large mixed echogenic mass in solid and cystic seen right adnexa measuring $14 \times 10 \mathrm{~cm}$. Computed tomography abdomen shows a large ovoid abdominopelvic mass measuring $10.3 \times 14.1 \times 14.8 \mathrm{~cm}$ solid and cystic components with intermingling calcification and fatty elements. There was enlarged right illac nodes. Diagnosis was malignant ovarian dermoid with torsion. (Fig.1) Routine haematological and biochemical parameters were within normal limits. Chest radiograph and electrocardiogram were within normal limits. Skeletal survey was normal The value of CA-125 was 56.65(normal<35U/ml). USG guided FNAC of ovarian mass shows benign cystic teratoma. A clinical diagnosis of benign cystic teratoma with malignancy was made and exploratory laparotomy was performed

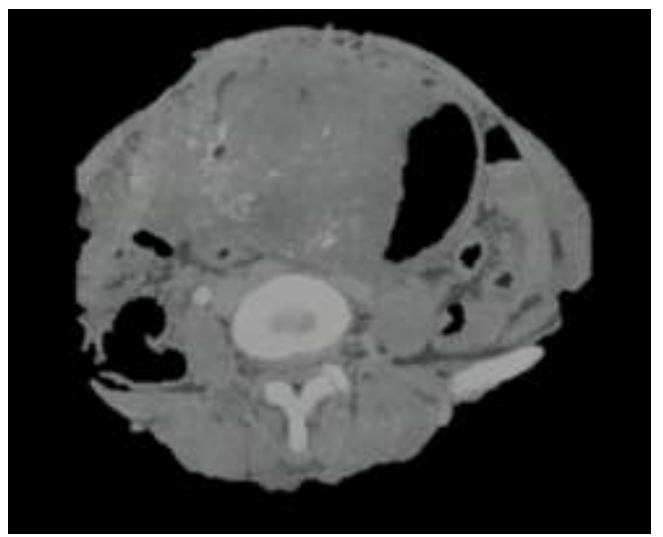

Fig. 1: CT shows a 10.3x14.1x14.8cm sized solid-cystic mass with intermingling calcification and fatty component in right ovary 
On entering the peritoneal cavity shows a irregular bosselated mass about $20 \times 15 \mathrm{~cm}$ arising from the right ovary. The tumour infiltrated along the right infundibulopelvic ligament upto the ovarian artery along with omental, pelvic and paraaortic lymph node involvement. Hysterectomy with bilateral salphingo-opherectomy, omentectomy, pelvic and para aortic lymph node dissection and peritoneal wash were performed. The postoperative was uneventful.

The resected specimen along with lymphnodes and peritoneal wash submitted for histopathological examination. Grossly the right ovarian tumour measured $15 \times 12 \times 6 \mathrm{~cm}$ and weighed $1300 \mathrm{gm}$. It was a multilocular. Cut surface shows multilocular solid and cystic mass with intact smooth capsule. The cut surface of right ovarian mass disclosed multiseptate myxoid and mucinous areas composed of hair tufts bone and keratin. Uterus, cervix, left tube were grossly unremarkable. Other ovary was normal.

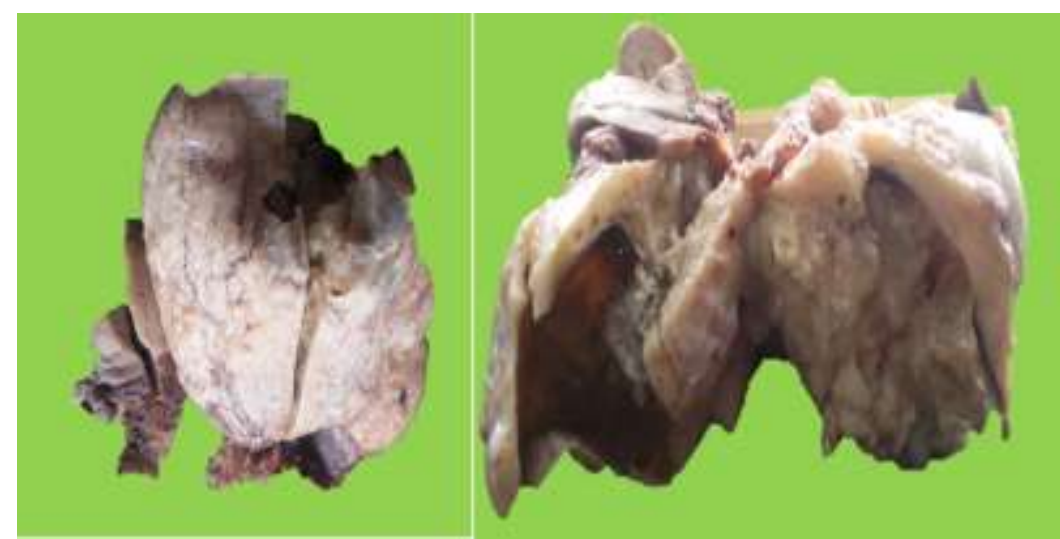

Fig.2: Macroscopic appearance of ovarian tumour shows multiseptated myxoid and mucinous areas composed of bone ,hair tufts admixed with keratin.

Microscopic evaluation of section demonstrate a mature cystic teratoma with mucinous adenocarcinoma arising from the right ovary. The mature cystic teratoma component consists of skin,bone and hair.

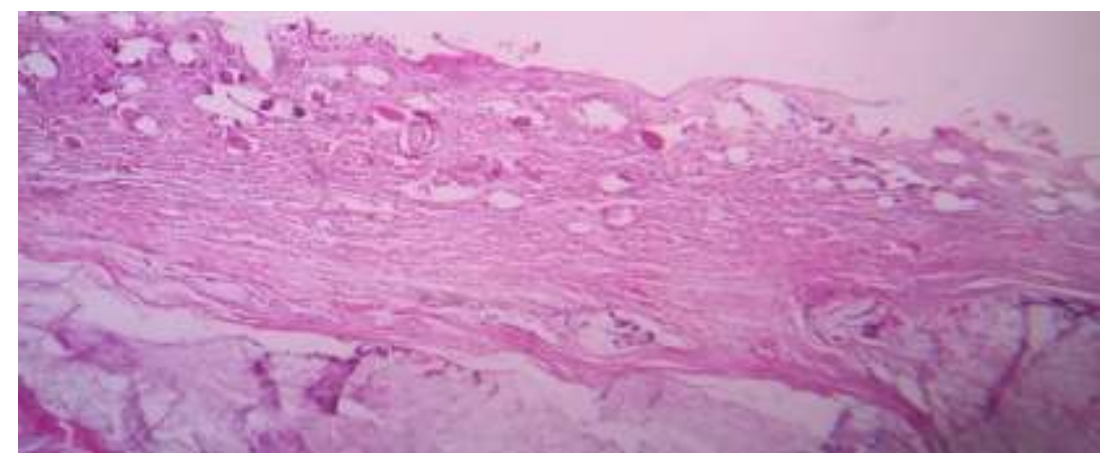

Fig.3: Microscopic appearance of cyst wall composed of ectodermal elements

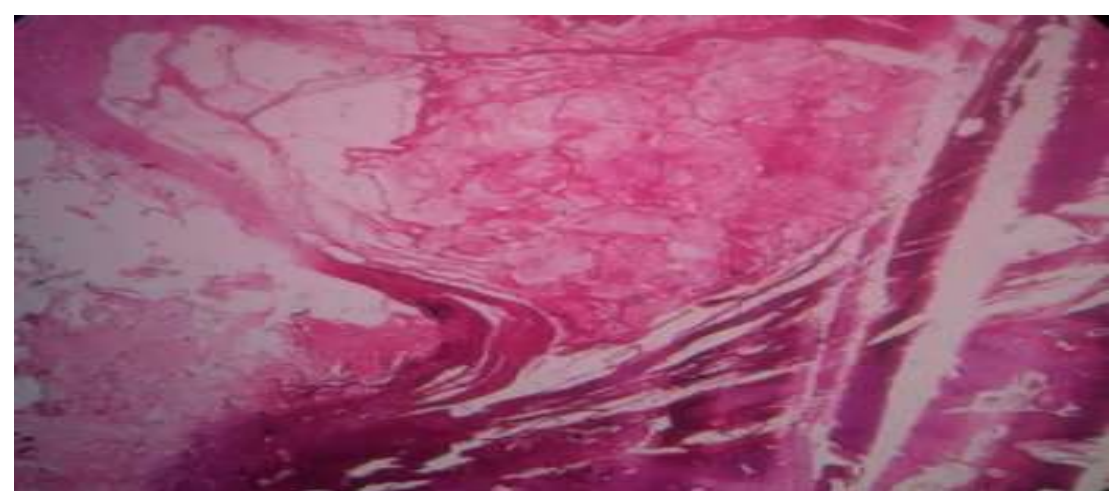

Fig.4: Section show mesodermal elements

The malignant component consist of mucinous adenoarcinoma composed of mucinous malignant epithelial cells infiltrating the parenchyma and the cells show signet ring cell appearance. 

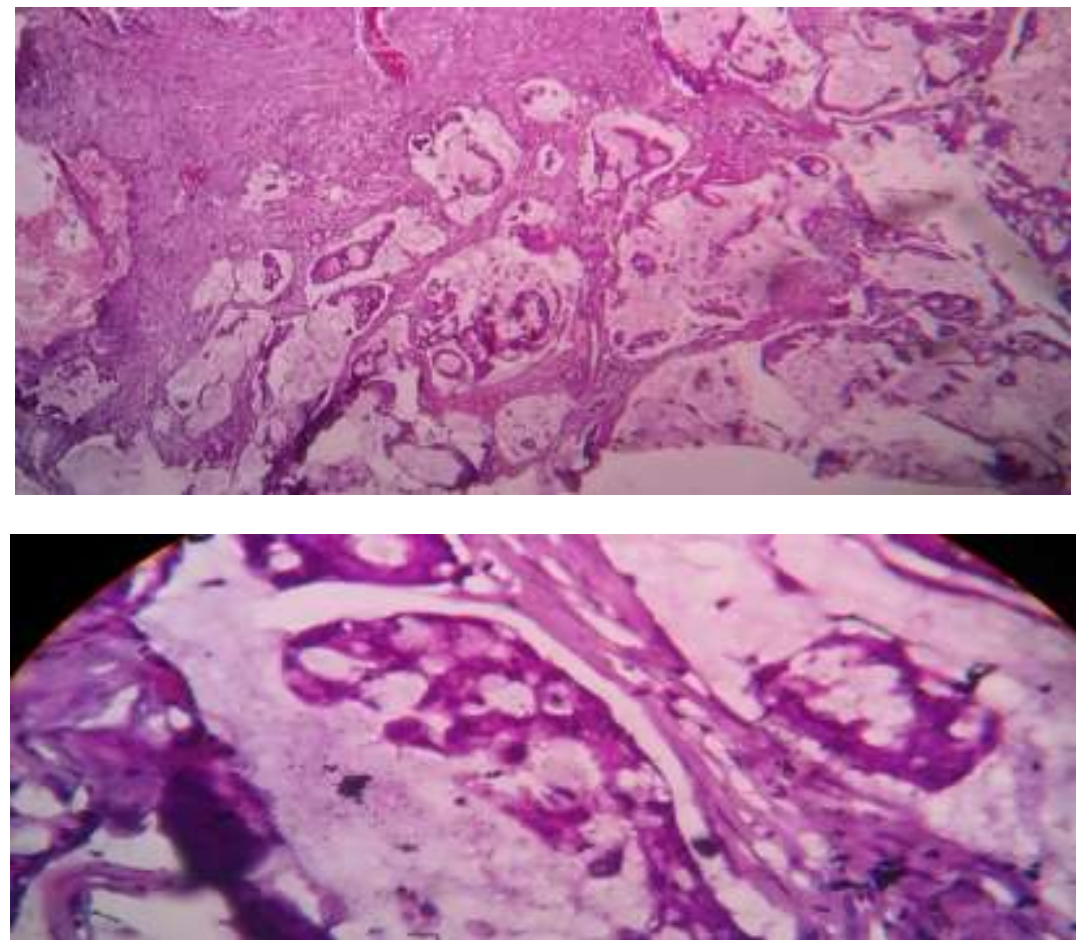

Fig.5: Section show mucinous adenocarcinoma composed of mucinous malignant epithelial cells with cells showing signet ring cell appearance.

Sections from the right tube, omental pelvic and para aortic nodes show tumour infiltration. Peritoneal cytology was positive for malignancy.

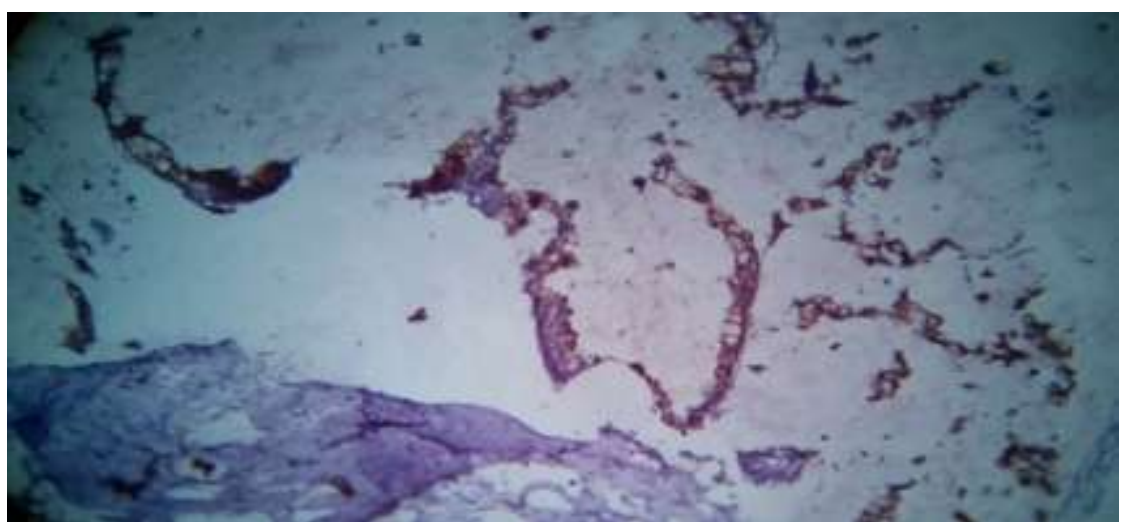

Fig.6: Immunohistochemistry for ck 20 positive

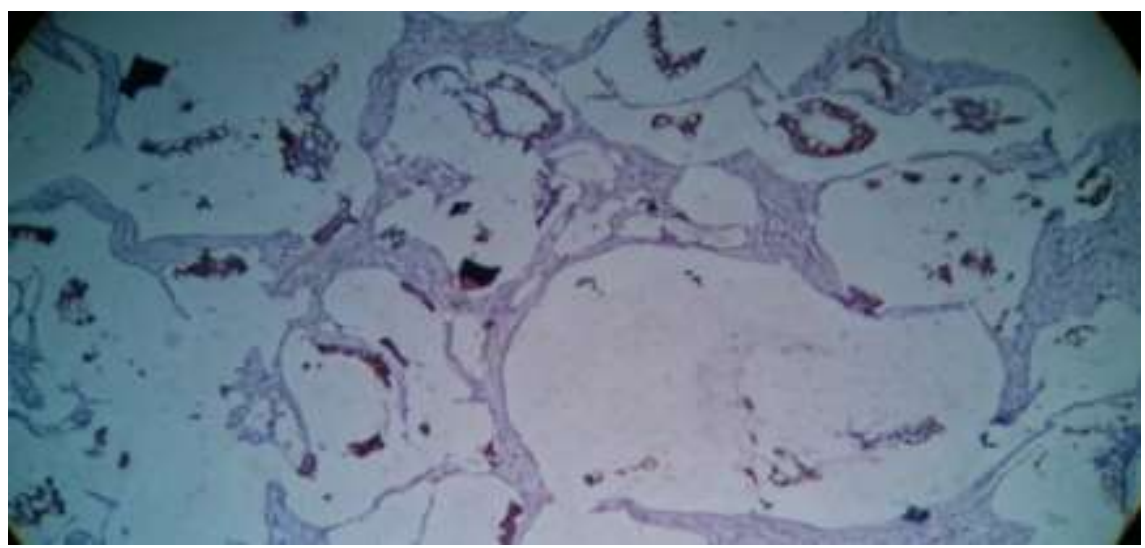

Fig.7: Immunohistochemistry CK 7 positive 


\section{Discussion}

The retrospective analysis on the spectrum of mucinous lesions of ovary at institute of Obstetrics and Gynaecology ,Govt hospital for Women and Children Chennai for a period of 5 years from January 2010-2014. Out of 350 ovarian tumors in the study period 19 cases (30\%) found to be mucinous adenocarcinoma of ovary. No case was associated with mature cystic teratoma.We present this case for its rarity and nodal metastasis.

Mature cystic teratomas (MCT) are recognized as one of the most common tumors in women during the reproductive age. Malignant change in benign cystic teratoma has been recorded as occurring in 1-3\% of cases. The most common form of malignant transformation of the MCT is squamous cell carcinoma. Other tumors arising in MCT include basal cell carcinoma, sebaceous tumor, malignant melanoma, adenocarcinoma, sarcoma, a neuroectodermal tumor. The risk of malignancy is related to age and is substantially greater in postmenopausal women, the highest incidence being in the fifth and sixth decades of life.

Malignant change is rarely recognized preoperatively. Most patients with such tumours have symptoms of abdominal pain and mass, which do not differ from those of uncomplicated mature cystic teratomas such as abdominal pain and mass. These tumours range in sizes from $30 \mathrm{~cm}$ to $40 \mathrm{~cm}$

Immuno histochemistry with ck 7 and ck20 were done to differentiate primary ovarian tumour with metastatic ovarian tumour.ck 7 and ck 20 were positive. Both positivity was reported in colorectal carcinoma and prostate carcinoma.

Patient was started on 3 cycles of chemotherapy with cisplatin, etoposide and bleomycin.

\section{Conclusion}

Mucinous adenocarcinoma arising from MCT with nodal metastasis is not widely reported. Studies have shown only 6-8\% of ovarian MCT is associated with mucinous tumours.

\section{References}

[1]. Griffiths D, Wass J, Look K, Sutton G. Malignant degeneration of a mature cystic teratoma five decades after discovery. GynecolOncol. 1995; 59:427-429. [PubMed]

[2]. Rose PG, Tak WK, Reale FR. Squamous cell carcinoma arising in a mature cystic teratoma with metastasis to the paraaortic nodes. GynecolOncol. 1993;50:131-133.[PubMed]

[3]. Krumerman MS, Chung A. Squamous carcinoma arising in benign cystic teratoma of the ovary: A report of four cases and review of the literature. Cancer. 1977;39:1237-1242. [PubMed]

[4]. Peterson WF: Malignant degeneration of benign cystic teratomas of the overy; a collective review of the literature. ObstetGynecolSurv 1957, 12: 793-830. 10.1097/00006254-195712000-00001PubMedView Article

[5]. Ueda G, Fujita M, Ogawa H, Sawada M, Inoue M, Tanizawa O: Adenocarcinoma in a benign cystic teratoma of the ovary: report of a case with a long survival period. GynecolOncol 1993, 48: 259-263. 10.1006/gyno.1993.1044PubMedView Article

[6]. Min KJ, Jee BC, Lee HS, Kim YB: Intestinal adenocarcinoma arising in a mature cystic teratoma of the ovary: a case report. Pathol Res Pract 2006, 202: 531-535. 10.1016/j.prp.2006.03.005PubMedView Article

[7]. Fishman A, Edelstein E, Altaras M, Beyth Y, Bernheim J: Adenocarcinoma arising from the gastrointestinal epithelium in benign cystic teratoma of the ovary. GynecolOncol 1998, 70: 418-420. 10.1006/gyno.1998.5123PubMedView Article 